\title{
Air-Water Interface Assembly of Protein Nanofibrils Promoted by Hydrophobic Additives
}

Lei Wang," Fredrik G. Bäcklund," Yusheng Yuan, Selvakumaran Nagamani, Piotr Hanczyc, Lech Sznitko, and Niclas Solin*

Cite This: ACS Sustainable Chem. Eng. 2021, 9, 9289-9299

Read Online

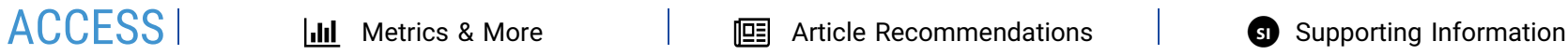

ABSTRACT: Herein we demonstrate a novel way of modifying the colloidal stability of proteins by the presence of hydrophobic molecules. A protein capable of self-assembly into protein nanofibrils (PNFs) is milled with a hydrophobic molecular material. Upon dissolution in acidic water followed by heating, the proteins are converted into PNFs containing hydrophobic dyes. When aqueous dispersions of such PNFs are heated, films are formed at the air-water interface. The films contain ordered, optically anisotropic domains, and the shape of the reaction vessel can influence the PNF packing. We demonstrate the generality of the process by employing PNFs derived from the three proteins bovine insulin (INS), $\beta$-lactoglobulin (BLG), and hen egg white lysozyme (HEWL) in combination with the dyes $\alpha$-sexithiophene (6T) and 4-(dicyanomethylene)-2-methyl-6-(4-dimethylaminostyryl)-

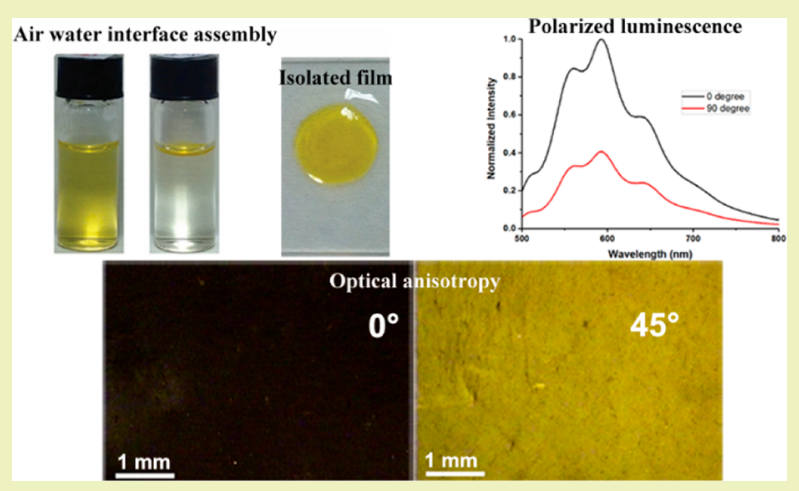
$4 H$-pyran (DCM). As individual dye molecules are oriented along the long PNF axis, whole ensembles of dyes become aligned and, as a result, display emission of polarized light. Moreover, in the case of PNFs stained with DCM, stimulated emission is demonstrated.

KEYWORDS: Protein nanofibrils, Mechanochemistry, Self-assembly, Luminescence, Laser dyes

\section{INTRODUCTION}

The development of materials from sustainable resources is a research area of increasing importance. As a wide variety of proteins can be isolated from natural sources and/or industrial waste streams, proteins can be a future source of sustainable materials. In combination with their rich supramolecular chemistry, this fact makes proteins promising candidates for the development of sustainable nanomaterials, possibly displaying emergent properties from the hierarchical organization. $^{1-4}$ An excellent candidate for the preparation of hierarchically assembled protein materials is amyloid fibrils., Although amyloid fibrils formed in vivo have traditionally been associated with diseases, there are now many examples of functional amyloids ${ }^{7,8}$ where organisms employ amyloid fibrils' attractive properties for constructive purposes: for example, as components in bacterial films ${ }^{9}$ or mussel adhesives. ${ }^{10}$ It has also been demonstrated that many proteins not related to diseases, ${ }^{8}$ including proteins from industrial waste streams, ${ }^{11}$ form amyloid-like structures in vitro. Hereafter we designate such fibrils as protein nanofibrils (PNFs). PNFs can be viewed as high-aspect-ratio rodlike particles, with lengths in the micrometer range and diameters of up to a few tens of nanometers. The mechanical properties of PNFs are outstanding, ${ }^{12}$ and their thermodynamic stability is high. ${ }^{13}$ As a consequence, PNFs are promising materials for future technological applications, ${ }^{14-18}$ especially as the preparation of PNFs is in general straightforward. ${ }^{19}$ A typical process is to heat the protein in acidic water (at a $\mathrm{pH}$ removed from the isoelectric point $(\mathrm{pI})$ of the protein), resulting in a readily processable aqueous PNF dispersion. ${ }^{20}$ However, it should be noted that for many proteins (especially for proteins of high molar mass) PNF formation is often accompanied by processes such as protein hydrolysis, meaning that the resulting dispersion is a mixture of PNFs and hydrolyzed protein fragments. $^{18,21}$ Thus, it is essential to develop processing techniques that give materials a proper function despite a significant amount of protein/peptide in the non-PNF form. Depending on physical variables such as concentration, $\mathrm{pH}$, ionic strength, and temperature, PNF dispersions can form gels and various liquid crystalline phases. ${ }^{14,22,23}$ Aqueous PNF dispersions accordingly display an impressive range of possibilities for processing into ordered materials. ${ }^{24}$ However, a limitation common to most proteins is the lack of

Received: March 19, 2021

Revised: June 21, 2021

Published: July 2, 2021 
chromophores (e.g., in the form of extended $\pi$-electron systems), limiting their possible application in organic electronics and photonics. For such applications, it is thus of interest to functionalize the PNF material: for example, by preparing materials modified with luminescent organic compounds. The majority of such compounds are highly hydrophobic due to the presence of a large number of aromatic rings, meaning that many such compounds have a low solubility even in organic solvents. This creates a practical mixing problem, as proteins typically are soluble in polar solvents where the solubility of many luminescent dyes is extremely low. We recently reported a method ${ }^{25-27}$ where the drawback of low solubility is turned into an advantage: by utilizing a mechanochemical approach, ${ }^{28}$ where dry powders of the protein and the hydrophobic compound are mixed in the solid state by grinding, hybrid materials are obtained that can be dissolved/dispersed in aqueous media. The hybrid materials undergo self-assembly into PNFs incorporating the hydrophobic molecules that may provide extra functionality to the resulting material, including luminescence $e^{25,26,29,30}$ or magnetic properties. $^{31}$

The physical features of proteins are a subtle balance between hydrophobicity and hydrophilicity, and an intriguing aspect is to modify the physical properties of PNF dispersions by hydrophobic molecules. The hydrophobic molecule could then act as both a functionalization agent and a hydrophobic additive. We have previously observed such effects; for example, in the case of hybrids between PNFs and a hydrophobic dye we found that protein spherulites with diameters up to $1 \mathrm{~mm}$ can be formed, in contrast to a typical diameter of $\sim 150 \mu \mathrm{m}$ in the absence of the hydrophobic dye. ${ }^{32}$ It is known that proteins and PNFs may assemble at the airwater interface, resulting in monolayers. ${ }^{33-36}$ Herein, we report that hydrophobically modified PNFs assemble at the air-water interface, resulting in multilayer PNF films containing optically anisotropic domains with sizes up to square centimeters. For hydrophobic materials, we employ two organic dyes. The first is $\alpha$-sexithiophene (6T), which is a highly hydrophobic molecule and has low solubility even in organic solvents. $6 \mathrm{~T}$ is a model commonly employed molecule in optoelectronics with the interesting property that its emission spectrum is very sensitive to the aggregation state of the molecule. ${ }^{37-39}$ The second hydrophobic organic dye presented in this study is 4-(dicyanomethylene)-2-methyl-6(4-dimethylaminostyryl)-4H-pyran (DCM), which is a widely employed red laser dye. ${ }^{40,41}$ In addition, we employ paraffins as examples of hydrophobic alkanes. We demonstrate that the methodology is general by employing three different proteins. We have used one low-molar-mass protein (insulin) displaying a high yield of PNF conversion (and consequently a low amount of protein/peptide in the non-PNF form) and two high-molar-mass proteins (lysozyme and $\beta$-lactoglobulin) displaying a moderate PNF conversion yield (that consequently contains a significant amount of hydrolyzed protein and peptides in the non-PNF form). We find that structurally ordered phases can also be formed for high-molar-mass proteins without removing proteins/peptides in the non-PNF form. As individual PNFs can align anisotropic dye molecules along the long fiber axis, the large area domain "nematic-like" phases contain ensembles of oriented dye molecules that display polarized light emission. The resulting layers can be isolated as freestanding films and can be readily transferred to various substrates. Films formed from PNFs functionalized with the laser dye DCM show stimulated emission, demonstrating that the functionalized PNF films can be used as matrices for mirrorless lasing.

\section{EXPERIMENTAL SECTION}

Materials. Bovine insulin (INS), hen egg-white lysozyme (HEWL), and $\beta$-lactoglobulin (BLG) were obtained from SigmaAldrich. $\alpha$-Sexithiophene (6T) and 4-(dicyanomethylene)-2-methyl6-(4-dimethylaminostyryl)-4H-pyran (DCM) were obtained from $\mathrm{TCl}$ and Exciton Inc., respectively. Paraffin was obtained from Kebo lab $A B$. All chemicals were used as received without further purification.

PNF Formation Procedure. In a typical procedure, the protein was milled with the hydrophobic dye in a 50:1 weight ratio. In all cases, the grinding process was carried out using a mortar and pestle for $10 \mathrm{~min}$. Next, the resulting hybrid materials were dissolved in 25 $\mathrm{mM} \mathrm{HCl}\left(\mathrm{pH} \mathrm{1.6)}\right.$ at a protein concentration of $5 \mathrm{mg} \mathrm{mL}^{-1}$ for INS and $10 \mathrm{mg} \mathrm{mL}^{-1}$ for BLG and HEWL. Then, solutions were heated at $65^{\circ} \mathrm{C}$ for $24 \mathrm{~h}$ (with stirring during the initial $1 \mathrm{~h}$ ) in the case of INS, at $80^{\circ} \mathrm{C}$ for $72 \mathrm{~h}$ (with continuous stirring) in the case of HEWL, and at $80{ }^{\circ} \mathrm{C}$ for $48 \mathrm{~h}$ in the case of BLG. After the allotted time, the resulting mixtures ware filtered by a $0.2 \mu \mathrm{m}$ PES membrane. Final PNF dispersions had protein concentrations equal to $5 \mathrm{mg} \mathrm{mL}^{-1}$ (INS) and $10 \mathrm{mg} \mathrm{mL}^{-1}$ (HEWL and BLG). We estimated the PNF conversion yield for the respective proteins by employing Amicon Ultra-15 centrifugal filter units. The estimations were carried out by comparing the mass of proteins collected on filters with the mass of proteins dissolved in examined solutions. For INS the yield was $96 \%$, for BLG $49 \%$, and for HEWL $34 \%$ with the UV-vis spectrophotometric measurement error in the range of ca. $5 \% .{ }^{42}$

Film Preparation. PNF films were fabricated in reaction vessels of two types: cylindrical vials with an inner diameter of $1 \mathrm{~cm}$ and rectangular containers with dimensions of $5.0 \times 4.8 \mathrm{~cm}$. The PNF dispersions were diluted to reach the desired protein concentration and then transferred to the reaction containers. The PNF dispersion was then left standing on a hot plate preheated to $80^{\circ} \mathrm{C}$ for up to $72 \mathrm{~h}$ before harvesting the films. For films formed from functionalized HEWL PNFs it was beneficial to perform film formation in the presence of salt $(30 \mathrm{mM} \mathrm{NaCl})$. The formed PNF films can be either transferred from the liquid onto substrates or isolated as freestanding films. Mechanically flexible freestanding films can be obtained by injecting $200 \mu \mathrm{L}$ of $10 \mathrm{mg} \mathrm{mL}^{-1}$ PVA solution into the container with the PNF film. After it was soaked overnight, the PVA-treated film was washed, transferred to a Parafilm piece, and dried. The film can then be peeled off from the Parafilm.

Atomic Force Microscopy. AFM measurements were performed using a Digital Instruments Dimension 3100 atomic force microscope. Samples were diluted 200 times from the reaction solution prior to applying to silica substrates and left to dry for $1 \mathrm{~min}$. Excess fluid was removed by applying a nitrogen gas flow. The contour length of more than 300 fibrils was counted, plotted, and fitted using a log-normal distribution to estimate the protein fibrils' length statistics.

Absorption and Fluorescence Spectroscopy. Absorption and transmittance spectra were obtained using a Shimadzu UV-2450 UVvis spectrometer. Fluorescence spectra were collected using a Horiba Jobin-Yvon Fluoromax-4 spectra fluorometer. The anisotropy of the $6 \mathrm{~T}$ orientation was measured as follows: samples were analyzed using a 405 or $450 \mathrm{~nm}$ laser as a light source and a polarizer mounted between the sample and the detector. A wavelength of $405 \mathrm{~nm}$ was used to excite the INS-6T-PNF film, while $450 \mathrm{~nm}$ was used for BLG6T-PNF and HEWL-6T-PNF films. The polarizing filter was turned $90^{\circ}$ relative to the sample between individual measurements, and the detector used a $450 \mathrm{~nm}$ long-pass filter. Data were collected from three different points on the film, and the mean values were plotted.

Polarized Light Microscopy. The polarized light microscopy images were recorded using an Olympus BH-2 microscope equipped with two polarizers, a rotatable sample stage, and a top-mounted digital camera. The large-area polarized light microscopy images were obtained by a stereo light microscope with a top-mounted digital 


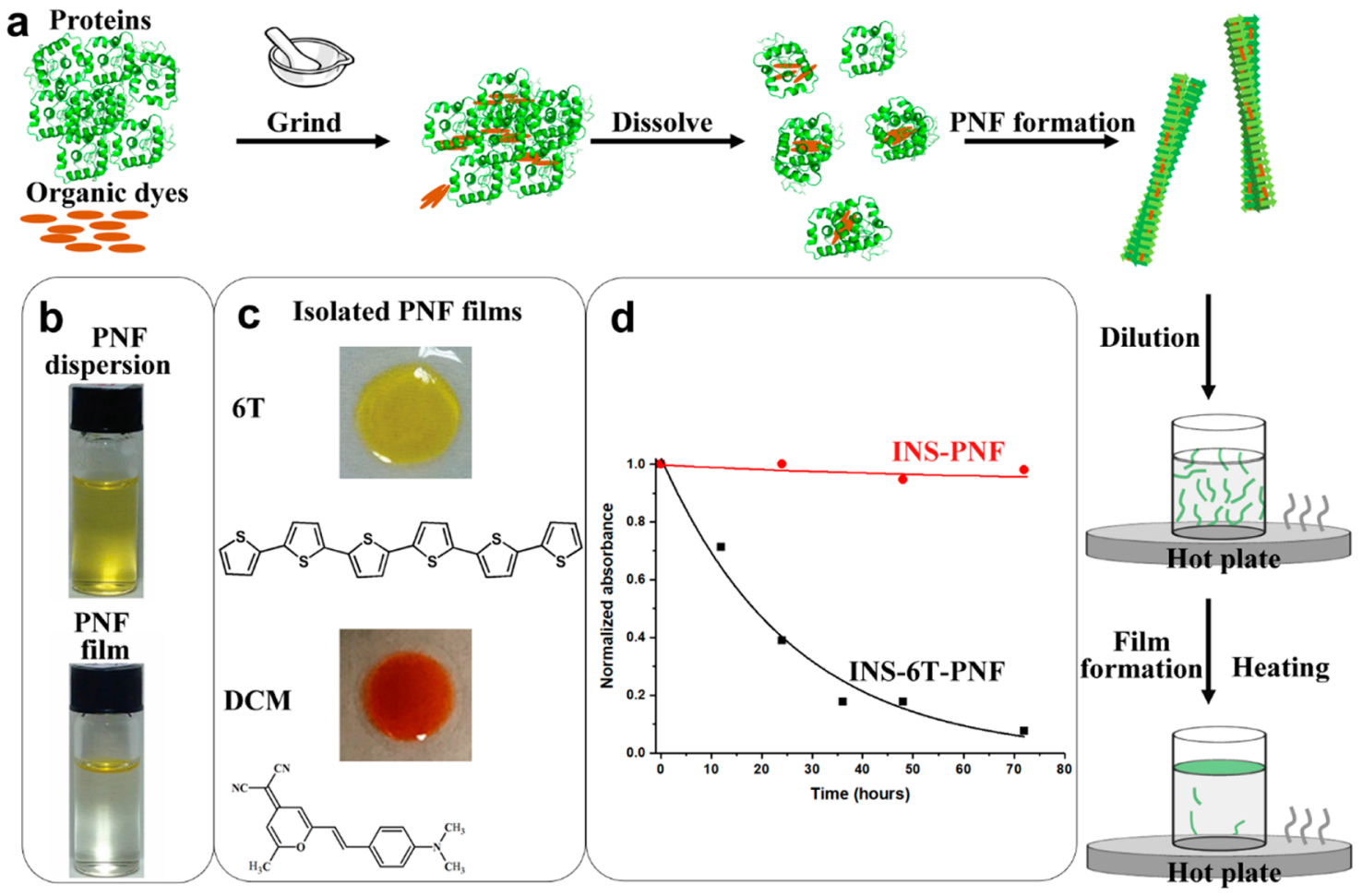

Figure 1. (a) Schematic drawing showing the five-step preparation of PNFs functionalized with hydrophobic dyes and preparation of the corresponding air-water interface PNF films. (b) Photographs of an INS-6T-PNF dispersion $\left(0.5 \mathrm{mg} \mathrm{mL}^{-1}\right)$ before and after the film formation at the air-water interface. (c) Photographs of films formed from 6T-PNFs and DCM-PNFs transferred to the glass slide as well as the chemical structures of 6T and DCM. (d) Absorbance measured as a function of time for film formation from an INS-6T-PNF dispersion $\left(0.5 \mathrm{mg} \mathrm{mL}^{-1}\right.$, in 25 $\mathrm{mM} \mathrm{HCl})$. For comparison, the absorbance measured for the INS-PNF dispersion $\left(0.5 \mathrm{mg} \mathrm{mL}^{-1}\right.$ in $\left.25 \mathrm{mM} \mathrm{HCl}\right)$ is shown.

camera modified by the addition of polarizers in a crossed polarizer setting.

Measurements of Film Thickness. Film thickness measurements were performed with a Dektak $6 \mathrm{M}$ stylus profiler equipped with a $12.5 \mu \mathrm{m}$ stylus tip from Veeco. Film thickness values were estimated by cutting the film with a scalpel and determining the maximum depth of the profile roughness below the mean line across such a cut.

Fluorescence Microscopy. The fluorescence microscope images were recorded with an epifluorescence microscope (Zeiss Axiovert inverted microscope A200 Mot) equipped with a CCD camera (Axiocam HR). Samples were excited at $405 \mathrm{~nm}$.

Mechanical Measurements. A Triboindenter TI-950 instrument with a Berkovich diamond indenter was used to perform nanoindentation measurements. For the determination of friction, a diamond conical tip ( $\sim 5 \mu$ m diameter) was employed.

Lasing Experimental Details. Lasing measurements were conducted using a $10 \mathrm{~Hz}$ Nd:YAG nanosecond pulse laser (Continuum, Surlite II), serving as a pumping source for the studied samples. For PNF samples stained with DCM, the excitation wavelength was $532 \mathrm{~nm}$ (6 ns pulse duration). The laser light was directed to the optical setup consisting of a focal beam expanding system and a cylindrical lens, converting the shape of the beam crosssection into a long and thin stripe in the area of sample excitation. The stripe length was set to $3 \mathrm{~mm}$, while the height was set to 0.5 $\mathrm{mm}$. Such a geometry supports the directionality of amplified light emission along the longer dimension of the exciting area. A fibercoupled high-resolution spectrometer (Andor Shamrock 163) equipped with a $1800 \mathrm{~mm} / 1$ grating was used (spectral resolution $0.1 \mathrm{~nm}$ ) to record emission spectra. The spectrograph was connected to a CCD camera (Andor iVac), enabling data acquisition. The fiber end (placed next to the edge of the sample) and the acquired optical signal were perpendicular to the pumping beam propagation direction. According to the Malus law concept, the control of exciting beam energy was provided by the rotation of a half-wave plate with respect to a Glan-Laser polarizer with a fixed polarization direction, both placed just after the Nd:YAG output coupler. The polarization of excitation light was vertical; thus, it was always perpendicular to the longer "stripe" dimension and expected STE direction.

\section{RESULTS AND DISCUSSION}

PNF and Film Formation Procedure. Formation of Functionalized PNFs. The entire procedure, from native protein to PNF film, involves five steps as outlined in Figure 1a. In a manner similar to previously reported procedures, ${ }^{26,29}$ the protein (bovine insulin (INS), $\beta$-lactoglobulin (BLG), or hen egg-white lysozyme (HEWL)) is milled with 2 wt \% of the hydrophobic dye/molecule ( $\alpha$-sexithiophene (6T), 4-(dicyanomethylene)-2-methyl-6-(4-dimethylaminostyryl)-4H-pyran (DCM), or paraffin), resulting in a powder mixture than can be dissolved in $25 \mathrm{mM} \mathrm{HCl}$. Upon heating with mechanical stirring, the functionalized protein is converted into functionalized PNFs. PNF formation for INS was performed at a protein concentration of $5 \mathrm{mg} \mathrm{mL}^{-1}$; for BLG and HEWL, the protein concentration was $10 \mathrm{mg} \mathrm{mL}^{-1}$. A discussion of the PNF formation process and absorption and fluorescence spectra and AFM images, including fibril length distributions, are available in the Supporting Information and Figures S1S5. The conversion yield for the different proteins was estimated by performing fibrillations of the unmodified proteins under the same conditions as for the functionalized proteins. For INS, the conversion yield was $96 \%$, for BLG $49 \%$, and for HEWL $34 \%$, and we assume the conversion yields to be roughly similar for the functionalized proteins. 
Formation of Functionalized PNF Films at the Air-Water Interface. When dispersions of functionalized PNFs at a suitable concentration are heated, films form at the air-water interface (Figure 1b) that can be isolated as freestanding films or put onto substrates and dried (Figure 1c). During film formation, the reaction containers were heated at $80{ }^{\circ} \mathrm{C}$ with a hot plate.

The kinetics of PNF film formation was monitored for an

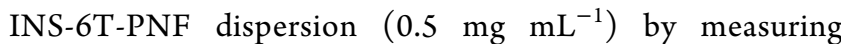
temporal changes at the absorption maximum positioned at the wavelength of $280 \mathrm{~nm}$ (Figure 1d). As can be seen, the decrease in absorption is an exponential process. After $72 \mathrm{~h}$, it reaches an almost constant value, indicating that the film's growth at the air-water is accomplished and little PNF material is left in the bulk liquid. As the PNF material is functionalized with $6 \mathrm{~T}$, it becomes yellow and the film formation is thus readily observable by the naked eye, as shown in Figure $1 \mathrm{~b}$. When film formation is attempted with a pristine INS-PNF dispersion (no hydrophobic additive and the same concentration of $\left.0.5 \mathrm{mg} \mathrm{mL}^{-1}\right)$, there is essentially no change in absorption after $72 \mathrm{~h}$, demonstrating that most of the protein remained in the sample's liquid phase (Figure 1d). This observation indicates that the presence of a hydrophobic additive promotes the film formation process at a low PNF concentration.

The functionalized PNF films can be isolated as freestanding films. However, these films were found to become brittle when they lose water, and the drying process leads to the formation of cracks. This feature makes working with freestanding films difficult, as they are prone to mechanical damage. The following procedure was employed to overcome the problem with brittleness: when the film formation process was finished, $200 \mu \mathrm{L}$ of a $10 \mathrm{mg} \mathrm{mL}^{-1}$ PVA solution was injected into the liquid containing the PNF film, which was then left overnight. Then, the PVA-treated film was washed and transferred to a Parafilm piece, and after drying, the film was peeled off. Such freestanding films gain enough plasticity to be conveniently handled.

Influence of Reaction Parameters on PNF Film Formation. PNF film formation was investigated with regard to five parameters: temperature, protein concentration, $\mathrm{pH}$, templating of premade films, and the shape of the reaction vessel. All of the parameters were critical for establishing a successful protocol for layer formation at the water-air interface.

Temperature. Heating is essential for PNF film formation at the air-water interface. Functionalized PNF dispersions can be stored for months at room temperature without any visible formation of films. It is, moreover, favorable to employ asymmetric heating in order to induce film formation. When film formation is attempted by symmetrical heating, for example by heating a vial with a PNF dispersion in an oil bath, film formation is irregular with poorer repeatability. The asymmetric heating generates a density difference in the liquid, leading to a convection flow that facilitates mass transport to the air-water interface and film formation at the surface.

Protein Concentration. Each of the utilized proteins has its critical concentration, above which PNF films can be formed. For the INS-6T-PNFs, we have found that the layer can be formed already at concentrations as low as $0.006 \mathrm{mg} \mathrm{mL}^{-1}$. However, for HEWL-6T-PNFs and BLG-6T-PNFs, it was necessary to employ a PNF concentration of at least $1 \mathrm{mg}$ $\mathrm{mL}^{-1}$ to ensure efficient film formation. If a concentration that is too low is employed, either no film is formed or incomplete films (that do not span the whole container) are formed. As a standard condition for forming films from functionalized HEWL and BLG PNFs, we employed a total protein concentration of $5 \mathrm{mg} \mathrm{mL}$. It should be noted that unmodified PNFs from HEWL and BLG also form films at the air-water interface at such concentrations.

$p H$. To determine how the balance of repulsive/attractive forces coming from fibril-fibril interactions affects the film formation process, we also tested the effect of $\mathrm{pH}$ on this process.

The $\mathrm{pH}$ of INS-6T-PNFs dispersions (at a concentration of $0.5 \mathrm{mg} \mathrm{mL}^{-1}$ ) was modified from 1.6 to either 4.0 or 7.0. Then the dispersions were heated on a hot plate for $72 \mathrm{~h}$, and the results were as follows. At $\mathrm{pH}$ 4.0, fibrils associate and form a gel located in the surface region (Figure 2a), whereas for film
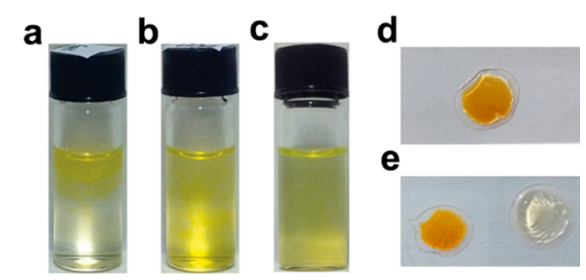

Figure 2. (a) INS-6T-PNFs $\left(0.5 \mathrm{mg} \mathrm{mL}^{-1}\right)$ at $\mathrm{pH} 4.0$ after $48 \mathrm{~h}$ of heating at $80^{\circ} \mathrm{C}$. (b) INS-6T-PNFs $\left(0.5 \mathrm{mg} \mathrm{mL}^{-1}\right)$ at $\mathrm{pH} 7.0$ after 48 $\mathrm{h}$ of heating at $80^{\circ} \mathrm{C}$. (c) A control sample showing INS-6T-PNFs $\left(0.5 \mathrm{mg} \mathrm{mL} \mathrm{m}^{-1}\right)$ at $\mathrm{pH} 4.0$ before heating. (d) An isolated film obtained from a vial where a HEWL paraffin PNF film was put at the air-water interface over a bulk liquid containing HEWL-6T-PNFs. The resulting film consists of two layers, with one layer built up from paraffin-PNF and the second from 6T-PNFs. (e) Isolated films obtained from a vial where a HEWL-paraffin-PNF film was put into the bulk liquid containing HEWL-6T-PNFs. The result was the formation of a 6T-PNF film at the air-water interface, while no growth occurred on the pre-existing paraffin-PNF film. The film formed at the air-water interface is shown to the left, and the preformed HEWL-paraffin-PNF film is shown to the right.

formation performed at $\mathrm{pH} 7.0$ a gel is formed that spans the whole reaction vessel (Figure $2 \mathrm{~b}$ ). These results illustrate the importance of a proper balance between repulsive and attractive interactions. If the fibril-fibril interaction is too strong, fibrils will interact in the bulk liquid, and film formation is prevented as the PNFs gets arrested in a gel state. Film formations from HEWL-6T-PNFs and BLG -6T-PNFs are less clear-cut. In both of these cases, films are still formed at $\mathrm{pH} 4.0$ and 7.0. However, under those conditions, simultaneously with film formation, PNFs agglomerates into small particles.

We have also tested the stability of premade films as a function of $\mathrm{pH}$. An example is provided for aged PVA-treated HEWL-6T-PNF films. The films were inserted into vials with water at different $\mathrm{pHs}(\mathrm{pH}-12)$ and photographed at the start $(0 \mathrm{~h})$ and after 5 days. The films are remarkably stable, and essentially no changes can be observed after 5 days (Figure S6).

Premade Films as Templates. In order to investigate the influence of premade PNF films and the importance of the air-water interface, we performed the following experiment. At first, we prepared two transparent freestanding films made of HEWL PNFs containing paraffin as a hydrophobic additive. Then we performed two experiments involving film formation 

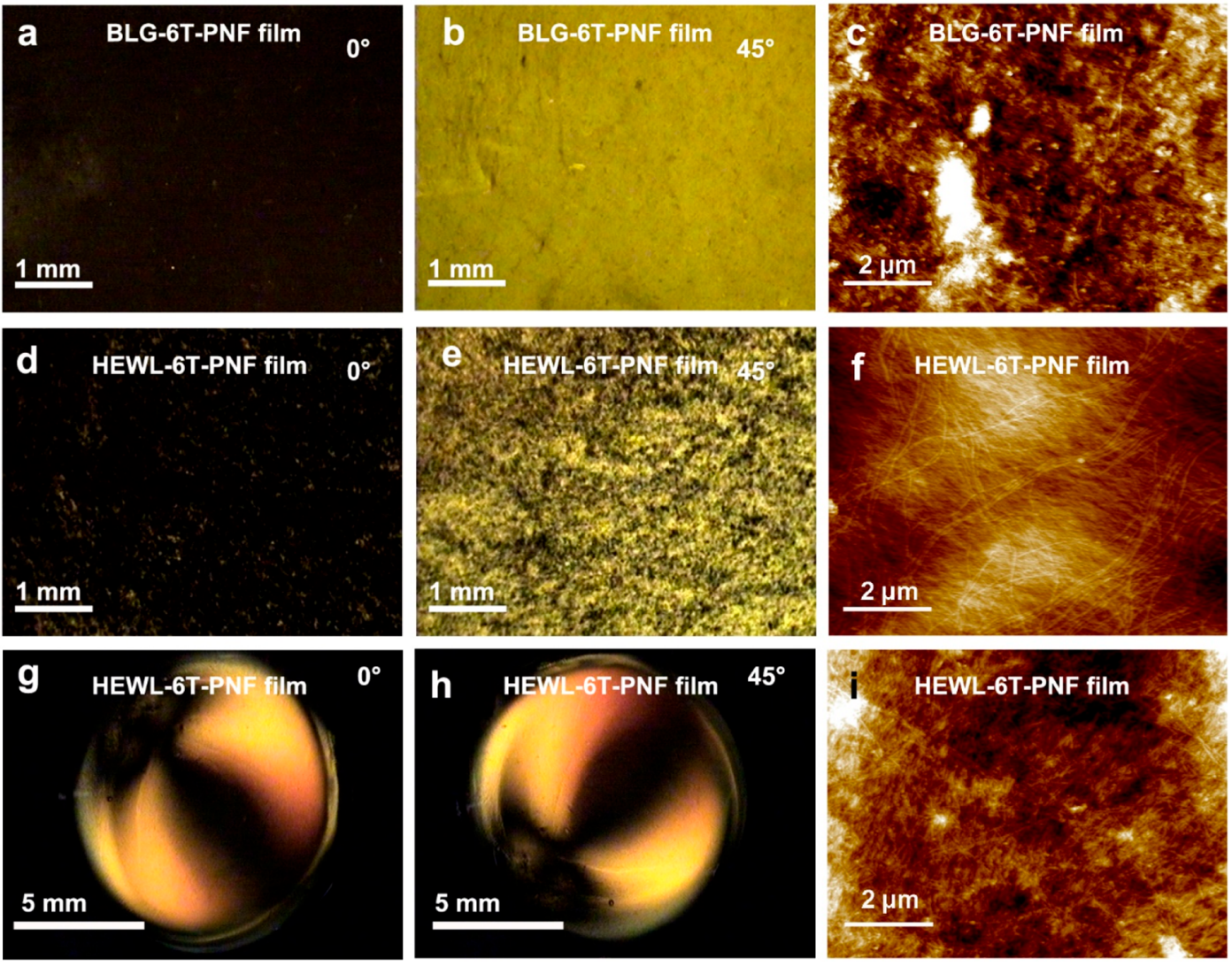

Figure 3. POM images and AFM images of films formed by air-water interface assembly. (a) POM image of a BLG-6T-PNF film (formed by airwater interface assembly in a square container) viewed with crossed polarizers. (b) The film shown in (a) turned by $45^{\circ}$ relative to the orientation in (a). (c) AFM image of a BLG-6T-PNF film formed in a square container. (d) POM image of a HEWL-6T-PNF film (formed by air-water interface assembly in a square container) viewed with crossed polarizers. (e) The film shown in (d) turned by $45^{\circ}$ relative to the orientation in (d). (f) AFM image of a HEWL-6T-PNF film formed in a square container.( g) POM image of a HEWL-6T-PNF film (formed by air-water interface assembly in a round container) viewed with crossed polarizers. (h) The film shown in (g) turned by $45^{\circ}$ relative to the orientation in (g). (i) AFM image of a HEWL-6T-PNF film formed in a round container.

of HEWL-6T-PNFs in the presence of the premade PNF films containing paraffin. In one case, the transparent film was placed at the air-water interface before the film formation procedure was initiated. In the second approach, the transparent film was placed in the bulk liquid. The results of this experiment are shown in Figure 2d,e, respectively. In the first case, the second film with 6T molecules has grown onto the transparent film kept at the air-water interface, forming a layer by layer structure. In the second case, the new film formed separately, at the air-water interface, rather than growing onto the film inserted into the bulk liquid. These results show that a premade film in the bulk liquid does not act as a nucleation point for film growth. It can thus be concluded that fibril-fibril interactions at the air-water interface are crucial for PNF film formation.

Shape of the Reaction Vessel. Another physical property that can be influenced during the film fabrication process is the arrangement of fibrils in the film. The shape of the container can highly influence the optical anisotropy of PNF films (Figure 3). The films formed in square containers tend to have one privileged orientation of fibrils, resulting in optically anisotropic domains, possibly forced by the reaction vessel's symmetry (see Figure $3 a-f$ and later discussion). On the other hand, films grown in round containers exhibit the fibrils' radial organization (see Figure $3 \mathrm{~g}-\mathrm{i}$ and later discussion).
Mechanical Properties of PNF Films. PNF films were isolated and dried on glass substrates. After drying, the thicknesses and mechanical properties were measured. Film thicknesses were found to range from $85 \mathrm{~nm}$ to $19 \mu \mathrm{m}$ (Table $\mathrm{S} 1$ ). A common material feature of all films is that the central region is thicker than the edge region. This difference may result from the convection flow pattern, which could be used to tune the film's morphology. As shown in Table S1, what is very important is that the resulting film thickness depends on the initial protein concentration and heating time. That makes the proposed protocol of layers formation very advantageous, as the thickness could be potentially controlled even at the nanometer scale.

The drying process of PNF films leads to the formation of cracks. The plasticity of films can be improved by soaking them in a PVA solution, as was described earlier. The mechanical properties of plasticized (with PVA) and unmodified films were investigated by nanoindentation measurements (Table S2). The resulting samples had elastic moduli between 2.80 and $4.33 \mathrm{GPa}$ and hardnesses between 0.26 and $0.38 \mathrm{GPa}$. In general, the films modified with PVA had a lower elastic modulus in comparison to unmodified samples. The results are in good agreement with measurements of the Young modulus of other reported protein fibrils. ${ }^{12,43}$

Optical Properties of PNF Films. Polarization Microscopy vs Morphology in Microscale. POM measurements were 

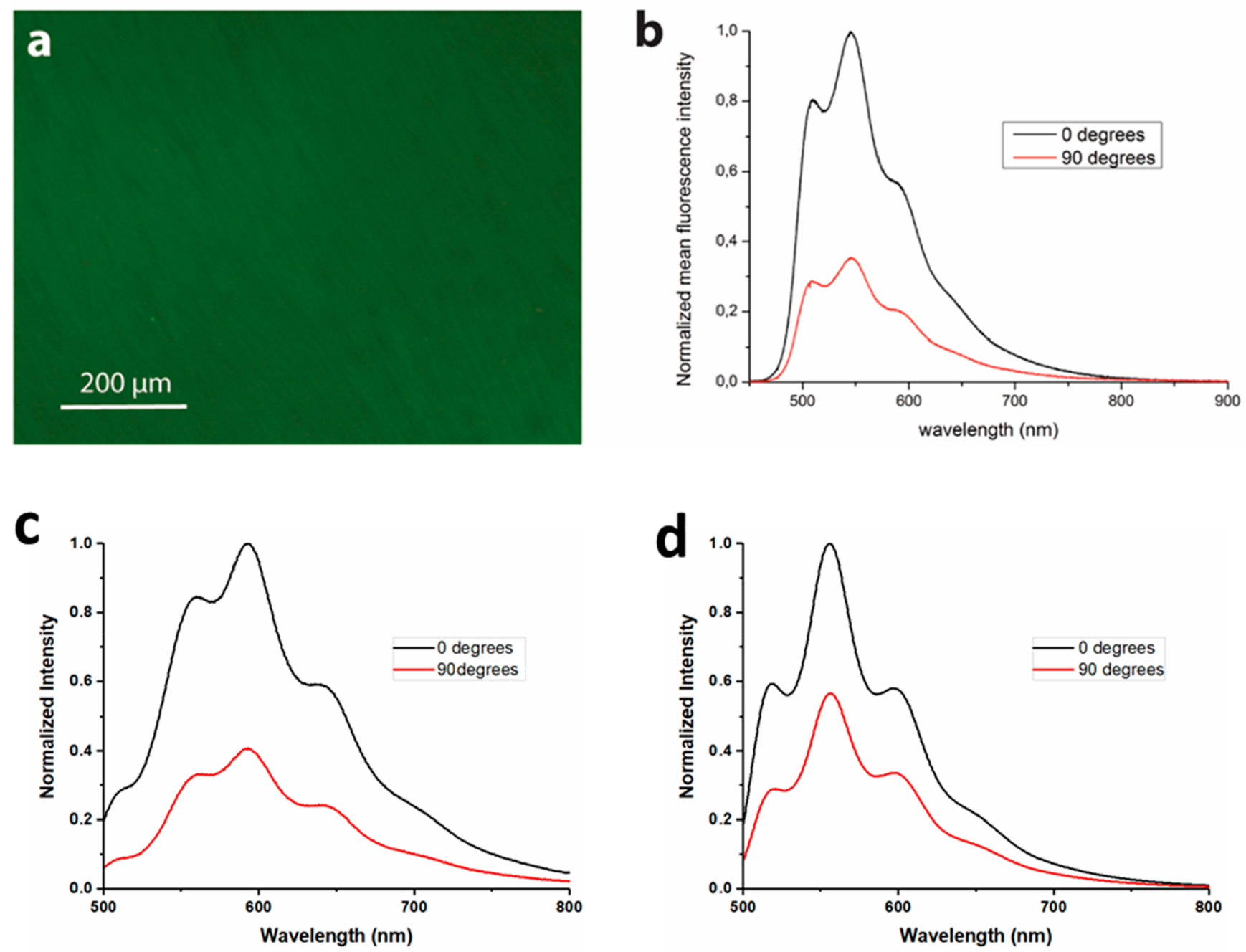

Figure 4. (a) Fluorescence microscope image of a dried INS-6T-PNF film. (b) Polarized emission from an INS-6T-PNF film. Altering the direction of a polarizer by $90^{\circ}$ relative to a film deposited at the stage will significantly alter the recorded emission intensity, indicating a high level of anisotropy for the fluorescent 6T molecules in the film. (c) BLG-6T-PNF film measured as described in (b). (d) HEWL-6T-PNF film measured as described in (b).

carried out for samples collected from the square and round containers and transferred to the glass substrates. Figure $3 \mathrm{a}, \mathrm{b}$ shows microscopy images of a BLG-6T-PNF film, collected from the square container, in two situations: the first when the film is rotated to crossed polarizers to exhibit the lowest light transparency (angle $0^{\circ}$; Figure $3 \mathrm{a}$ ) and the second when the film is rotated by an angle of $45^{\circ}$, which refers to the highest light transparency through the crossed-polarizers. This result indicates that the films contain optically anisotropic domains, with an ordered internal structure and some disordered/ isotropic regions (dark spots). HEWL-6T-PNF films display similar behavior but with smaller dimensions of the anisotropic domains (Figure $3 \mathrm{~d}, \mathrm{e}$ ). The local microscopic structure of the PNF film surface (films prepared from BLG-6T-PNFs and HEWL-6T-PNFs) was visualized by AFM, as shown in Figure 3.

As examples of films formed in round containers, POM and AFM images of a HEWL-6T-PNF film are shown in Figure $3 \mathrm{~g}-\mathrm{i}$. Also, films formed in round containers display clear birefringence; however, the patterns are entirely different from those formed in square containers. For films formed in round containers, both bright and dark regions are visible in the film regardless of the sample's orientation relative to the polarizers. We surmise that these patterns stem from radially oriented fibrils that would generate crosslike patterns similar to those observed in POM images of polymer and protein spherulites. $^{32,44,45}$

Figures $S 7$ and $S 8$ show additional POM images from INS6T-PNF, HEWL-6T-PNF, and BLG-6T-PNF films formed in square and round containers, respectively. Data for films from PNFs incorporating DCM and paraffin are shown in Figures S9 and S10. All of these films show the same features as the films displayed in Figure 3.

Luminescence Anisotropy of PNF Films Doped with 6T Dye. The luminescent properties of PNF films were investigated for samples composed of INS, HEWL, and BLG proteins, doped with $6 \mathrm{~T}$ dye. Each film was harvested from a square container, placed on a glass substrat, and left for drying. Such prepared samples were studied using fluorescence microscopy and UV-vis fluorescence spectroscopy. An example of a fluorescence microscope image of an INS-6TPNF film on a glass slide is shown in Figure 4a. The sample was excited at $405 \mathrm{~nm}$, which resulted in green fluorescence due to the presence of $6 \mathrm{~T}$ molecules. Moreover, it has been 
(a)

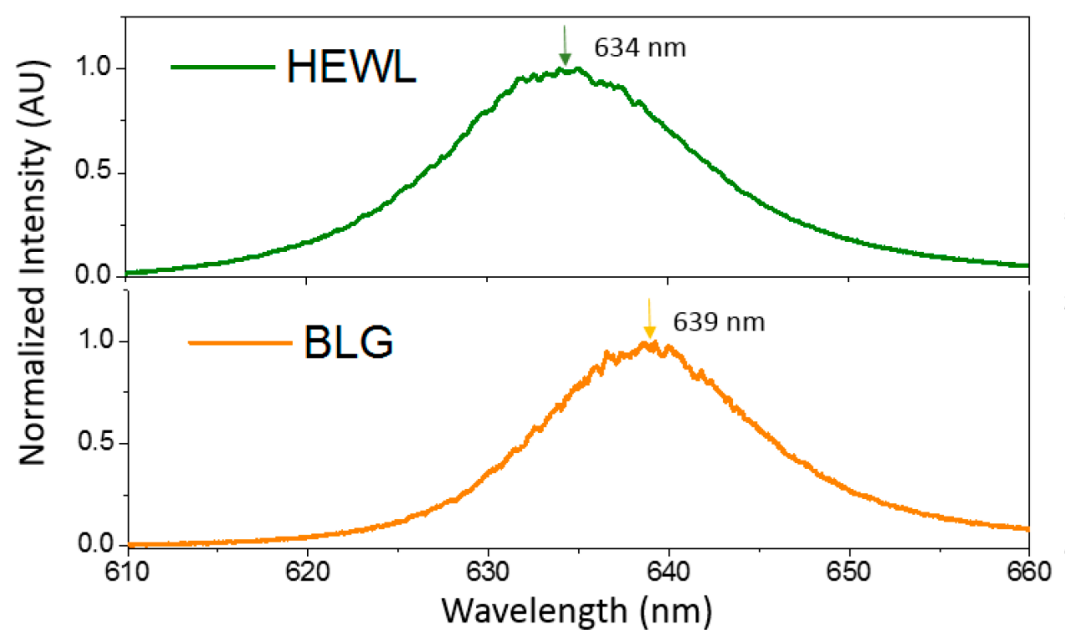

(b)

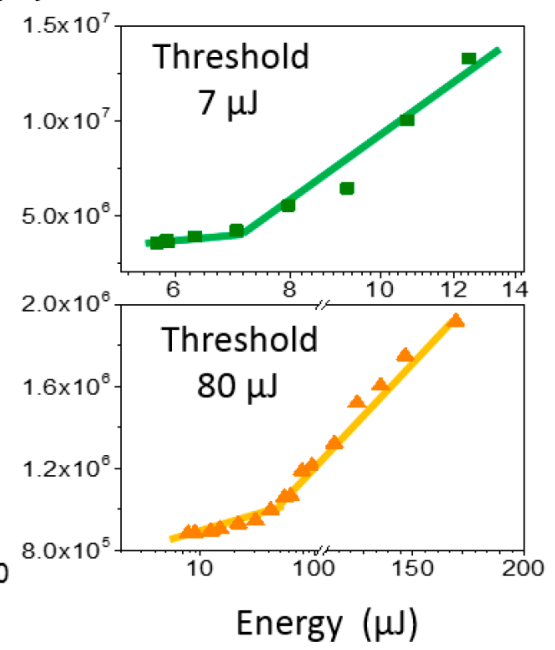

Figure 5. (a) Stimulated emission (STE) spectra in freestanding films of HEWL-DCM-PNFs (green) and BLG-DCM-PNFs (orange). (b) STE thresholds of HEWL-DCM-PNF films (green) and BLG-DCM-PNF films (orange).

demonstrated that PNFs can orient linear flat aromatic compounds along the long fibril axis. ${ }^{26}$ This means that the anisotropic arrangement of PNFs is likely to be transferred to the incorporated dye molecules. This was probed for a film prepared from INS-6T-PNFs by utilizing a polarization filter that was turned $90^{\circ}$ relative to the sample between individual measurements. Typical spectra are shown in Figure $4 b-d$. The high degree of anisotropy of the air-water interface assembled INS-6T-PNF film resulted in a 70\% difference in emission intensity for perpendicular orientations of polarization. Three areas of the sample were analyzed, and all measurements showed similar results. The films of BLG-6T-PNFs and HEWL-6T-PNFs also show a high degree of anisotropy. A $60 \%$ difference in emission intensity was observed for BLG-6TPNFs (Figure 4c), whereas for the HEWL-6T-PNF film, this parameter was equal to $44 \%$. The trends for the different proteins regarding the polarization degree of emitted light match the trend observed for PNF conversion yields (INS > BLG > HEWL).

Mirrorless Lasing from PNF Films Doped with DCM Dye. Due to the high price of bovine insulin, we focused our study on the low-cost proteins HEWL and BLG to demonstrate the application of "green" materials in optical lasing. DCM-PNF films (prepared from HEWL-DCM-PNFs and BLG-DCMPNFs) formed at the air-water interface display absorbance and fluorescence spectra similar to those of the corresponding PNF dispersions in water (see Figures S11 and S12 for absorbance and fluorescence spectra for films). Such films were examined as possible materials involving applications as mirrorless lasers. In brief, the lasing process occurs when photons emitted spontaneously by excited molecules are multiplied in the stimulated emission (STE) process caused by the interaction with other excited molecules during their propagation through the medium. The result is a directional and enhanced emission of light with a spectrum significantly narrower than that of fluorescence generated by spontaneous emission. A prerequisite for STE is the population inversion in the light-amplifying molecules. DCM is reported as a lasing molecule whereby the light amplification occurs in the fourlevel energy system ${ }^{46,47}$ when dye molecules are dissolved (for example, in the polymer matrix).
Figure 5 represents STE spectra and STE thresholds of HEWL-DCM-PNF and BLG-DCM-PNF films. The films were removed from the air-water interface and dried for the STE experiments. In the case of HEWL-DCM-PNFs, the STE occurs at $634 \mathrm{~nm}$ and for BLG-DCM-PNFs at $639 \mathrm{~nm}$. The emission maximum is red-shifted by $30 \mathrm{~nm}$ in comparison to the monomer's emission recorded in the polymer matrix at 606 $\mathrm{nm} .{ }^{46}$ The red shift that appears in DCM-PNFs may be related to the dye molecule organization along the oriented PNFs in the nematic phase. DCM dyes may become aligned in the PNF grooves, forming structures resembling J-type aggregates. ${ }^{48}$ Emission amplification was observed with increasing energy fluence, where the DCM STE threshold for the process was estimated to be $7 \mu \mathrm{J}$ (approximately $0.5 \mathrm{~mJ} / \mathrm{cm}^{2}$ ) for HEWLDCM-PNFs and $80 \mu \mathrm{J}$ (approximately $5.3 \mathrm{~mJ} / \mathrm{cm}^{2}$ ) for BLGDCM-PNFs. The results are comparable to results obtained for DCM dispersed in standard polymeric matrices. ${ }^{46,49}$ The protein films employed herein are thus an interesting alternative for matrix materials in organic solid-state lasers.

Biopolymeric Materials in Optoelectronics and Photonics: The Potential of PNFs. A wide variety of biopolymeric materials have been investigated for applications in photonics, ${ }^{50-58}$ with two prominent examples being nanocelluloses ${ }^{59-61}$ and DNA. ${ }^{62-64}$ One important challenge is to translate the rich information content of biopolymers at the molecular level into structural order at the macroscopic scale. ${ }^{65}$ From this aspect, DNA and nanocelluloses have their respective strong points. Nanocelluloses are mechanically strong and have a high aspect ratio and can thus be assembled into films built up from ordered nanocellulose fibrils; however, a drawback is that individual fibers do not contain suitable binding sites for dyes. On the other hand, DNA contains a range of binding sites and can accordingly readily be functionalized with dyes; however, DNA is relatively mechanically weak, and it is challenging to form ordered macroscopic materials from DNA. In comparison to DNA and nanocellulose, PNFs have good mechanical properties and can be easily functionalized with dyes. There is a growing interest in using PNFs in optoelectronics and photonics ${ }^{16,24,66-73}$ because of the richness of the protein materials and their generic structural features of the PNF state. ${ }^{8,18}$ Individual 
PNFs contain structurally ordered arrays of $\beta$-sheets presenting hydrophobic grooves along the long fiber axis. In addition, the high aspect ratio of PNFs leads to a strong tendency for PNFs to form ordered liquid crystalline phases, with PNFs aligned in one direction. ${ }^{18}$ This means that structural order on the nanometer length scale can be linked to structural order at the mesoscale. The commonly accepted model for amyloid-dye interactions is that the dye binds to the hydrophobic grooves present in the PNF structure. ${ }^{18,26}$ Anisotropic dyes thus typically bind with their long axis parallel to the long fibril axis. This in turn means that for a structure built up from ordered PNFs that order will be transferred to the ensemble of dyes, allowing for anisotropic optical properties.

The presented results herein show that PNFs can organize dye molecules, and the methodology employing sequential film growth can be used to fabricate layered structures of square centimeter size, having potential relevance in modern optoelectronics and photonics. Many optoelectronic and photonic devices such as light-emitting diodes, organic light detectors, lasers, etc. ${ }^{74-76}$ frequently exhibit layer by layer structures.

The methodology presented in this article allows for the easy preparation of advanced luminescent materials that are spontaneously formed by the self-assembly of proteins at the air-water interface. As the precursor materials are prepared simply by dry milling of powders followed by heating of the acidic aqueous protein dispersion, the methodology is readily amenable to scale-up. To the best of our knowledge, this is the first time that a mechanochemical methodology has been combined with self-assembly in this manner. The generality of the methodology is demonstrated by employing three different proteins. INS is employed as a convenient model protein, due to its attractive fibrillation characteristics; however, INS is not suitable for practical applications due to its high cost. On the other hand, HEWL is significantly cheaper than INS and is readily available in large quantities; HEWL is, for example, extensively employed as a food preservative in the cheese industry (with E number E1105). Finally, BLG is a component of whey protein, which is a side product during cheese production. BLG has been extensively investigated for a range of novel applications. ${ }^{77}$ In addition to the proteins investigated herein, many other proteins isolated on a large scale, often as industrial waste materials, have been shown to form $\mathrm{PNFs}^{11,78-80}$ and may potentially form ordered materials in a manner similar to that of the materials described herein. The methodology described above thus conforms with many of the 12 principles of green chemistry ${ }^{81}$ and presents a convenient route to fabricate "green" nanomaterials with a hierarchical structural order at multiple length scales. In addition, as the air-water interface is soft and dynamic, it is likely that the film formation process may be perturbed by different stimuli (vibrations, acoustic waves, heat gradients, ...), allowing for tuning of the PNF packing and thus systematic modification of structural and optical properties of PNF assemblies.

\section{CONCLUSIONS}

To summarize, we have developed a novel methodology for the formation of functionalized PNF films and demonstrated the generality of the method by employing three different proteins (insulin (INS), $\beta$-lactoglobulin (BLG), and lysozyme (HEWL)) and two dyes ( $\alpha$-sexithiophene (6T) and 4(dicyanomethylene)-2-methyl-6-(4-dimethylaminostyryl)-4Hpyran (DCM)). We have shown that the presence of hydrophobic additives promotes film formation and that these hydrophobic molecules can add properties to the PNF films; for example, by employing luminescent dyes to PNF, the films become luminescent. Moreover, the films are built up from domains with aligned PNFs, as manifested by birefringence during observations by polarized light microscopy. In addition, PNFs contain binding sites where anisotropic dyes are oriented with their long axis along the long fiber axis, and as a result, the ensemble of dye molecules shows net alignment and hence the emission of polarized light. Parameters such as film thickness can be controlled by the concentration of proteins in the PNF dispersion and/or the reaction time. Moreover, the shape of the container employed during the film formation process strongly influences the optical anisotropy of PNF films, demonstrating the flexibility of the procedure. From an operational perspective, the procedure employed to obtain these relatively advanced nanomaterials simply involves heating of a liquid in a container and is similar to that employed in preparing, e.g., tofu skin. ${ }^{82,83}$

The amount of readily available hydrophobic molecules is tremendous, and a wide range of proteins are known to form PNFs. Thus, the approach presented herein should be possible to apply, with small adjustments, for a wide range of systems and applications. Films can, for example, be prepared to contain hydrophobic drug molecules, pesticides, or nutrients. The limiting factor for the employed materials is that they must be stable under the conditions used for PNF formation. The approach of modifying the colloidal and self-assembly behavior may also be applicable to other protein systems, biopolymers, or other molecules undergoing self-assembly. By milling a soluble self-assembling molecule with an insoluble molecule, hybrids are obtained that, upon dispersion in liquid, may show novel self-assembly behavior. Apart from valorizing the biopolymer, the presence of the hydrophobic material may enable both the formation of novel structures and function. The presented methodology is thus extremely flexible with regard to both applicable materials and processing details and should accordingly be a valuable addition to the rapidly growing toolbox of "green" methodology for the materials scientist. $^{84,85}$

\section{ASSOCIATED CONTENT}

Supporting Information

The Supporting Information is available free of charge at https://pubs.acs.org/doi/10.1021/acssuschemeng.1c01901.

AFM images, fluorescence spectra, and absorption spectra of dye-doped PNFs, polarized optical microscopy images, and test of the chemical stability of the dyedoped PNF films (PDF)

\section{AUTHOR INFORMATION}

\section{Corresponding Author}

Niclas Solin - Department of Physics, Chemistry, and Biology, Electronic and Photonic Materials, Biomolecular and Organic Electronics, Linköping University, Linköping 581 83, Sweden; (1) orcid.org/0000-0002-0915-2575; Email: niclas.solin@liu.se

\section{Authors}

Lei Wang - Department of Physics, Chemistry, and Biology, Electronic and Photonic Materials, Biomolecular and Organic Electronics, Linköping University, Linköping 581 83, Sweden 
Fredrik G. Bäcklund - Department of Physics, Chemistry, and Biology, Electronic and Photonic Materials, Biomolecular and Organic Electronics, Linköping University, Linköping 581 83, Sweden

Yusheng Yuan - Department of Physics, Chemistry, and Biology, Electronic and Photonic Materials, Biomolecular and Organic Electronics, Linköping University, Linköping 581 83, Sweden

Selvakumaran Nagamani - Department of Physics, Chemistry, and Biology, Electronic and Photonic Materials, Biomolecular and Organic Electronics, Linköping University, Linköping 581 83, Sweden; Present Address: Sustainable Energy and Smart Materials Research Lab, Department of Nanoscience and Technology, Alagappa University, Karaikudi, Tamil Nadu, 630 003, India

Piotr Hanczyc - Department of Physics, Chemistry, and Biology, Electronic and Photonic Materials, Biomolecular and Organic Electronics, Linköping University, Linköping 581 83, Sweden; Institute of Experimental Physics, Faculty of Physics, University of Warsaw, 02-093 Warsaw, Poland; (1) orcid.org/0000-0002-1460-8477

Lech Sznitko - The Advanced Materials Engineering and Modelling Group, Wroclaw University of Science and Technology, 50-370 Wroclaw, Poland; (1) orcid.org/00000002-7013-7999

Complete contact information is available at: https://pubs.acs.org/10.1021/acssuschemeng.1c01901

\section{Author Contributions}

"L.W. and F.G.B. contributed equally.

\section{Notes}

The authors declare no competing financial interest.

\section{ACKNOWLEDGMENTS}

L.W. and Y.Y. acknowledge support from the China Scholarship Council (CSC), N.S. and S.N. acknowledge support from the Carl Tryggers foundation, and P.H. acknowledges support from the Polish National Agency for Academic Exchange Bekker programme. We acknowledge Rui Shu for assistance with nanoindentation measurements. N.S. acknowledges support from the Swedish Government Strategic Research Area in Materials Science on Functional Materials at Linköping University (Faculty Grant SFO-Mat-LiU No. 2009-00971).

\section{REFERENCES}

(1) Lash, M. H.; Fedorchak, M. V.; McCarthy, J. J.; Little, S. R. Scaling up self-assembly: bottom-up approaches to macroscopic particle organization. Soft Matter 2015, 11, 5597-5609.

(2) Fratzl, P.; Weinkamer, R. Nature's hierarchical materials. Prog. Mater. Sci. 2007, 52, 1263-1334.

(3) Pan, N. Exploring the significance of structural hierarchy in material systems-A review. Appl. Phys. Rev. 2014, 1, 021302.

(4) Kushner, A. M.; Guan, Z. B. Modular Design in Natural and Biomimetic Soft Materials. Angew. Chem., Int. Ed. 2011, 50, 90269057.

(5) Chiti, F.; Dobson, C. M. Protein misfolding, functional amyloid, and human disease. Annu. Rev. Biochem. 2006, 75, 333-366.

(6) Knowles, T. P.; Vendruscolo, M.; Dobson, C. M. The amyloid state and its association with protein misfolding diseases. Nat. Rev. Mol. Cell Biol. 2014, 15, 384-396.

(7) Fowler, D. M.; Koulov, A. V.; Balch, W. E.; Kelly, J. W. Functional amyloid - from bacteria to humans. Trends Biochem. Sci. 2007, 32, 217-224.
(8) Shen, Y.; Levin, A.; Kamada, A.; Toprakcioglu, Z.; RodriguezGarcia, M.; Xu, Y.; Knowles, T. P. J. From Protein Building Blocks to Functional Materials. ACS Nano 2021, 15, 5819-5837.

(9) Vlamakis, H.; Chai, Y. R.; Beauregard, P.; Losick, R.; Kolter, R. Sticking together: building a biofilm the Bacillus subtilis way. Nat. Rev. Microbiol. 2013, 11, 157-168.

(10) Zhong, C.; Gurry, T.; Cheng, A. A.; Downey, J.; Deng, Z. T.; Stultz, C. M.; Lu, T. K. Strong underwater adhesives made by selfassembling multi-protein nanofibres. Nat. Nanotechnol. 2014, 9, 858866.

(11) Josefsson, L.; Ye, X.; Brett, C. J.; Meijer, J.; Olsson, C.; Sjögren, A.; Sundlöf, J.; Davydok, A.; Langton, M.; Emmer, Å.; Lendel, C. Potato Protein Nanofibrils Produced from a Starch Industry Sidestream. ACS Sustainable Chem. Eng. 2020, 8, 1058-1067.

(12) Knowles, T. P. J.; Buehler, M. J. Nanomechanics of functional and pathological amyloid materials. Nat. Nanotechnol. 2011, 6, 469479.

(13) Balchin, D.; Hayer-Hartl, M.; Hartl, F. U. In vivo aspects of protein folding and quality control. Science 2016, 353, aac4354.

(14) Cherny, I.; Gazit, E. Amyloids: not only pathological agents but also ordered nanomaterials. Angew. Chem., Int. Ed. 2008, 47, 40624069.

(15) Li, D.; Jones, E. M.; Sawaya, M. R.; Furukawa, H.; Luo, F.; Ivanova, M.; Sievers, S. A.; Wang, W. Y.; Yaghi, O. M.; Liu, C.; Eisenberg, D. S. Structure-Based Design of Functional Amyloid Materials. J. Am. Chem. Soc. 2014, 136, 18044-18051.

(16) Hauser, C. A. E.; Maurer-Stroh, S.; Martins, I. C. Amyloidbased nanosensors and nanodevices. Chem. Soc. Rev. 2014, 43, 53265345.

(17) Sznitko, L.; Hanczyc, P.; Mysliwiec, J.; Samoc, M. Lowthreshold stimulated emission from lysozyme amyloid fibrils doped with a blue laser dye. Appl. Phys. Lett. 2015, 106, 023702.

(18) Wei, G.; Su, Z.; Reynolds, N. P.; Arosio, P.; Hamley, I. W.; Gazit, E.; Mezzenga, R. Self-assembling peptide and protein amyloids: from structure to tailored function in nanotechnology. Chem. Soc. Rev. 2017, 46, 4661-4708.

(19) Ye, X.; Lendel, C.; Langton, M.; Olsson, R. T.; Hedenqvist, M. S. Protein nanofibrils: Preparation, properties, and possible applications in industrial nanomaterials 2019, 29-63.

(20) Cao, Y.; Mezzenga, R. Design principles of food gels. Nature Food 2020, 1, 106-118.

(21) Ye, X.; Hedenqvist, M. S.; Langton, M.; Lendel, C. On the role of peptide hydrolysis for fibrillation kinetics and amyloid fibril morphology. RSC Adv. 2018, 8, 6915-6924.

(22) Corrigan, A. M.; Muller, C.; Krebs, M. R. H. The formation of nematic liquid crystal phases by hen lysozyme amyloid fibrils. J. Am. Chem. Soc. 2006, 128, 14740-14741.

(23) Jung, J. M.; Mezzenga, R. Liquid crystalline phase behavior of protein fibers in water: experiments versus theory. Langmuir 2010, 26, 504-514.

(24) Knowles, T. P. J.; Oppenheim, T. W.; Buell, A. K.; Chirgadze, D. Y.; Welland, M. E. Nanostructured films from hierarchical selfassembly of amyloidogenic proteins. Nat. Nanotechnol. 2010, 5, 204207.

(25) Rizzo, A.; Inganas, O.; Solin, N. Preparation of phosphorescent amyloid-like protein fibrils. Chem. - Eur. J. 2010, 16, 4190-4195.

(26) Bäcklund, F. G.; Wigenius, J.; Westerlund, F.; Inganäs, O.; Solin, N. Amyloid fibrils as dispersing agents for oligothiophenes: control of photophysical properties through nanoscale templating and flow induced fibril alignment. J. Mater. Chem. C 2014, 2, 7811-7822.

(27) Wang, L.; Solin, N. Preparation of functionalized protein materials assisted by mechanochemistry. J. Mater. Sci. 2018, 53, 13719-13732.

(28) James, S. L.; Friscic, T. Mechanochemistry. Chem. Soc. Rev. 2013, 42, 7494-7496.

(29) Bäcklund, F. G.; Solin, N. Development and application of methodology for rapid screening of potential amyloid probes. ACS Comb. Sci. 2014, 16, 721-729. 
(30) Hanczyc, P.; Procyk, M.; Radzewicz, C.; Fita, P. Two-photon excited lasing of Coumarin 307 for lysozyme amyloid fibrils detection. J. Biophotonics 2019, 12, e201900052.

(31) Andersson, B. V.; Skoglund, C.; Uvdal, K.; Solin, N. Preparation of amyloid-like fibrils containing magnetic iron oxide nanoparticles: effect of protein aggregation on proton relaxivity. Biochem. Biophys. Res. Commun. 2012, 419, 682-686.

(32) Bäcklund, F. G.; Solin, N. Tuning the aqueous self-assembly process of insulin by a hydrophobic additive. RSC Adv. 2015, 5, 92254-92262.

(33) Li, S.; Leblanc, R. M. Aggregation of Insulin at the Interface. J. Phys. Chem. B 2014, 118, 1181-1188.

(34) Noskov, B. A.; Bykov, A. G.; Gochev, G.; Lin, S. Y.; Loglio, G.; Miller, R.; Milyaeva, O. Y. Adsorption layer formation in dispersions of protein aggregates. Adv. Colloid Interface Sci. 2020, 276, 102086.

(35) Jordens, S.; Isa, L.; Usov, I.; Mezzenga, R. Non-equilibrium nature of two-dimensional isotropic and nematic coexistence in amyloid fibrils at liquid interfaces. Nat. Commun. 2013, 4, 1917.

(36) Isa, L.; Jung, J.-M.; Mezzenga, R. Unravelling adsorption and alignment of amyloid fibrils at interfaces by probe particle tracking. Soft Matter 2011, 7, 8127-8134.

(37) Fichou, D. Structural order in conjugated oligothiophenes and its implications on opto-electronic devices. J. Mater. Chem. 2000, 10, $571-588$.

(38) Ohno, S.; Tanaka, H.; Tanaka, K.; Takahashi, K.; Tanaka, M. Sexithiophene ultrathin films on passivated $\mathrm{Si}(001)$ surfaces: Growth and electronic structure. Org. Electron. 2015, 25, 170-177.

(39) Loi, M. A.; da Como, E.; Dinelli, F.; Murgia, M.; Zamboni, R.; Biscarini, F.; Muccini, M. Supramolecular organization in ultra-thin films of $\alpha$-sexithiophene on silicon dioxide. Nat. Mater. 2004, 4, 8185.

(40) Alcaire, M.; Cerdan, L.; Zamarro, F. L.; Aparicio, F. J.; Gonzalez, J. C.; Ferrer, F. J.; Borras, A.; Espinos, J. P.; Barranco, A. Multicolored Emission and Lasing in DCM-Adamantane Plasma Nanocomposite Optical Films. ACS Appl. Mater. Interfaces 2017, 9, 8948-8959.

(41) Sarkar, A.; Ojha, N. N. S.; Bhaktha, B. N. S. Effect of photonic stop-band on the modes of a weakly scattering DCM-PVA waveguide random laser. Appl. Phys. Lett. 2017, 110, 251104.

(42) Sooväli, L.; Rõõm, E.-I.; Kütt, A.; Kaljurand, I.; Leito, I. Uncertainty sources in UV-Vis spectrophotometric measurement. Accredit. Qual. Assur. 2006, 11, 246-255.

(43) Simone Ruggeri, F.; Habchi, J.; Cerreta, A.; Dietler, G. AFMBased Single Molecule Techniques: Unraveling the Amyloid Pathogenic Species. Current pharmaceutical design 2016, 22, 39503970.

(44) Krebs, M. R. H.; MacPhee, C. E.; Miller, A. F.; Dunlop, I. E.; Dobson, C. M.; Donald, A. M. The formation of spherulites by amyloid fibrils of bovine insulin. Proc. Natl. Acad. Sci. U. S. A. 2004, 101, 14420-14424.

(45) Shtukenberg, A. G.; Punin, Y. O.; Gunn, E.; Kahr, B. Spherulites. Chem. Rev. 2012, 112, 1805-1838.

(46) Sznitko, L.; Cyprych, K.; Szukalski, A.; Miniewicz, A.; Mysliwiec, J. Coherent-incoherent random lasing based on nanorubbing induced cavities. Laser Phys. Lett. 2014, 11, 045801.

(47) Tahir, M.; Sayyad, M. H.; Said, S. M.; Mohd Sabri, M. F.; Sarker, M. R. Amplified spontaneous emission and optical gain characteristics of sexithiophene single crystals. Opt. Mater. 2020, 100, 109695.

(48) Hanczyc, P.; Sznitko, L.; Zhong, C.; Heeger, A. J. Stimulated Emission from Rhodamine 6G Aggregates Self-Assembled on Amyloid Protein Fibrils. ACS Photonics 2015, 2, 1755-1762.

(49) Wan, Y.; Deng, L. Recyclable coherent random lasers assisted by plasmonic nanoparticles in DCM-PVA thin films. Opt. Express 2019, 27, 27103-27111.

(50) Shan, D.; Gerhard, E.; Zhang, C.; Tierney, J. W.; Xie, D.; Liu, Z.; Yang, J. Polymeric biomaterials for biophotonic applications. Bioactive materials 2018, 3, 434-445.
(51) Xiong, R.; Luan, J.; Kang, S.; Ye, C.; Singamaneni, S.; Tsukruk, V. V. Biopolymeric photonic structures: design, fabrication, and emerging applications. Chem. Soc. Rev. 2020, 49, 983-1031.

(52) Jacucci, G.; Schertel, L.; Zhang, Y.; Yang, H.; Vignolini, S. Light Management with Natural Materials: From Whiteness to Transparency. Advanced materials 2020, 2001215.

(53) Nyström, G.; Mezzenga, R. Liquid crystalline filamentous biological colloids: Analogies and differences. Curr. Opin. Colloid Interface Sci. 2018, 38, 30-44.

(54) Fresta, E.; Fernández-Luna, V.; Coto, P. B.; Costa, R. D. Merging Biology and Solid-State Lighting: Recent Advances in LightEmitting Diodes Based on Biological Materials. Adv. Funct. Mater. 2018, 28, 1707011.

(55) Cavinato, L. M.; Fresta, E.; Ferrara, S.; Costa, R. D. Merging Biology and Photovoltaics: How Nature Helps Sun-Catching. Adv. Energy Mater. 2021, 2100520.

(56) Borkner, C. B.; Elsner, M. B.; Scheibel, T. Coatings and films made of silk proteins. ACS Appl. Mater. Interfaces 2014, 6, 1561115625 .

(57) Mysliwiec, J.; Cyprych, K.; Sznitko, L.; Miniewicz, A. Biomaterials in light amplification. J. Opt. 2017, 19, 033003.

(58) Sznitko, L.; Mysliwiec, J.; Miniewicz, A. The role of polymers in random lasing. J. Polym. Sci., Part B: Polym. Phys. 2015, 53, 951-974.

(59) Eichhorn, S. J. Cellulose nanofibres for photonics and plasmonics. Current Opinion in Green and Sustainable Chemistry 2018, 12, 1-7.

(60) Lagerwall, J. P. F.; Schütz, C.; Salajkova, M.; Noh, J.; Hyun Park, J.; Scalia, G.; Bergström, L. Cellulose nanocrystal-based materials: from liquid crystal self-assembly and glass formation to multifunctional thin films. NPG Asia Mater. 2014, 6, e80.

(61) De France, K.; Zeng, Z.; Wu, T.; Nystrom, G. Functional Materials from Nanocellulose: Utilizing Structure-Property Relationships in Bottom-Up Fabrication. Adv. Mater. 2020, 2000657.

(62) Steckl, A. J. DNA - a new material for photonics? Nat. Photonics 2007, 1, 3-5.

(63) Grote, J. G.; Diggs, D. E.; Nelson, R. L.; Zetts, J. S.; Hopkins, F. K.; Ogata, N.; Hagen, J. A.; Heckman, E.; Yaney, P. P.; Stone, M. O.; Dalton, L. R. DNA Photonics [Deoxyribonucleic Acid]. Mol. Cryst. Liq. Cryst. 2005, 426, 3-17.

(64) Gomez, E. F.; Venkatraman, V.; Grote, J. G.; Steckl, A. J. Exploring the Potential of Nucleic Acid Bases in Organic Light Emitting Diodes. Adv. Mater. 2015, 27, 7552-7562.

(65) Liddle, J. A.; Gallatin, G. M. Nanomanufacturing: A Perspective. ACS Nano 2016, 10, 2995-3014.

(66) Herland, A.; Thomsson, D.; Mirzov, O.; Scheblykin, I. G.; Inganäs, $\mathrm{O}$. Decoration of amyloid fibrils with luminescent conjugated polymers. J. Mater. Chem. 2008, 18, 126-132.

(67) Barrau, S.; Zhang, F.; Herland, A.; Mammo, W.; Andersson, M. R.; Inganäs, O. Integration of amyloid nanowires in organic solar cells. Appl. Phys. Lett. 2008, 93, 023307.

(68) Acar, H.; Garifullin, R.; Aygun, L. E.; Okyay, A. K.; Guler, M. $\mathrm{O}$. Amyloid-like peptide nanofiber templated titania nanostructures as dye sensitized solar cell anodic materials. J. Mater. Chem. A 2013, 1, 10979.

(69) Bolisetty, S.; Adamcik, J.; Heier, J.; Mezzenga, R. Amyloid Directed Synthesis of Titanium Dioxide Nanowires and Their Applications in Hybrid Photovoltaic Devices. Adv. Funct. Mater. 2012, 22, 3424-3428.

(70) Rizzo, A.; Solin, N.; Lindgren, L. J.; Andersson, M. R.; Inganas, O. White light with phosphorescent protein fibrils in OLEDs. Nano Lett. 2010, 10, 2225-2230.

(71) Tanaka, H.; Herland, A.; Lindgren, L. J.; Tsutsui, T.; Andersson, M. R.; Inganäs, O. Enhanced Current Efficiency from Bio-Organic Light-Emitting Diodes Using Decorated Amyloid Fibrils with Conjugated Polymer. Nano Lett. 2008, 8, 2858-2861.

(72) Solin, N.; Inganäs, O. Protein Nanofibrils Balance Colours in Organic White-Light-Emitting Diodes. Isr. J. Chem. 2012, 52, 529539. 
(73) Apter, B.; Fainberg, B.; Handelman, A.; Lapsker, I.; Accardo, A.; Diaferia, C.; Morelli, G.; Rosenman, G. Long-Range Fluorescence Propagation in Amyloidogenic $\beta$-Sheet Films and Fibers. Adv. Opt. Mater. 2020, 8, 2000056.

(74) Yokota, T.; Zalar, P.; Kaltenbrunner, M.; Jinno, H.; Matsuhisa, N.; Kitanosako, H.; Tachibana, Y.; Yukita, W.; Koizumi, M.; Someya, T. Ultraflexible organic photonic skin. Science advances 2016, 2, e1501856.

(75) Baeg, K. J.; Binda, M.; Natali, D.; Caironi, M.; Noh, Y. Y. Organic light detectors: photodiodes and phototransistors. Adv. Mater. 2013, 25, 4267-4295.

(76) Foucher, C.; Guilhabert, B.; Kanibolotsky, A. L.; Skabara, P. J.; Laurand, N.; Dawson, M. D. RGB and white-emitting organic lasers on flexible glass. Opt. Express 2016, 24, 2273-2280.

(77) Cao, Y.; Mezzenga, R. Food protein amyloid fibrils: Origin, structure, formation, characterization, applications and health implications. Adv. Colloid Interface Sci. 2019, 269, 334-356.

(78) Agnieray, H.; Glasson, J. L.; Chen, Q.; Kaur, M.; Domigan, L. J. Recent developments in sustainably sourced protein-based biomaterials. Biochem. Soc. Trans. 2021, 49, 953-964.

(79) Peydayesh, M.; Mezzenga, R. Protein nanofibrils for next generation sustainable water purification. Nat. Commun. 2021, 12, 3248.

(80) Herneke, A.; Lendel, C.; Johansson, D.; Newson, W.; Hedenqvist, M.; Karkehabadi, S.; Jonsson, D.; Langton, M. Protein Nanofibrils for Sustainable Food-Characterization and Comparison of Fibrils from a Broad Range of Plant Protein Isolates. ACS Food Science \& Technology 2021, 1, 854.

(81) Zuin, V. G.; Eilks, I.; Elschami, M.; Kümmerer, K. Education in green chemistry and in sustainable chemistry: perspectives towards sustainability. Green Chem. 2021, 23, 1594-1608.

(82) Wu, L. C.; Bates, R. P. Soy protein-lipid films. 1. Studies on the film formation phenomenon. J. Food Sci. 1972, 37, 36-39.

(83) Chen, Y. M.; Ono, T. The Mechanisms for Yuba Formation and Its Stable Lipid. J. Agric. Food Chem. 2010, 58, 6485-6489.

(84) Ates, B.; Koytepe, S.; Ulu, A.; Gurses, C.; Thakur, V. K. Chemistry, Structures, and Advanced Applications of Nanocomposites from Biorenewable Resources. Chem. Rev. 2020, 120, 93049362.

(85) Percebom, A. M.; Towesend, V. J.; de Paula Silva de Andrade Pereira, M.; Pérez Gramatges, A. Sustainable self-assembly strategies for emerging nanomaterials. Current Opinion in Green and Sustainable Chemistry 2018, 12, 8-14. 